\title{
The effect of the non-target object position on wrist motion
}

\author{
Jun Li, Azwirman Gusrialdi, Sandra Hirche and Anna Schuboe
}

\begin{abstract}
In the present work we show the important role of wrist movements for the avoidance of non-target objects (obstacles) in a psychological pick-and-place experiment. The results show that humans can adjust the position of their wrist as well as the angle between their underarm and their hand during a prehension movement relative to the position of a non-target object. This indicates that humans tend to grasp the target object from the side which is further away from the non-target object. Based on these results, a preliminary model is proposed to simulate the wrist movement with respect to the position of a non-target object. We introduce this model with regard to a possible application in human-robot interaction and to better understand human's collision avoidance mechanism.
\end{abstract}

Keyword: wrist movement $\cdot$ grasping $\bullet$ obstacle avoidance • control model $\bullet$ pick-and-place

\section{INTRODUCTION}

$\mathrm{H}$ umans have the remarkable ability to flexibly adjust their arm and hand posture during grasping in order to avoid possible collisions with potential non-target objects. Jeannerod [1] proposed two independent visuomotor components in order to describe the transport of the hand (reach component) and the size of the grip (grasp component) during prehensile movements. Following this theory, many studies have shown that both components can be altered in the presence of a non-target object (see [2] [3] [4] [5]). For example, the movement time of the hand is increased when a non-target object was present in the workspace [2]. This result shows that a grasping movement can be adjusted on-line in the presence of a non-target object. Moreover, Mark Mon-Williams et al. [3] observed that the movement time is dependent on the position of the non-target object when this object is positioned on the left or right side of the target object in the same horizontal and vertical distance. For example, when the non-target object was located on the right side of the target object, there was a large effect in movement time. In addition, it was shown that the hand adjustment is significantly different for different positions of the non-target object. It was also reported that there is a different deviation

J. Li and A. Schuboe are with the Ludwig-Maximilian-University Munich, Germany (e-mail: jun.li@psy.lmu.de, anna.schuboe@lmu.de ).

A. Gusrialdi and S. Hirche are with the Institute of Automatic Control Engineering, Technical University of Munich, Germany (email: iman@1sr.ei.tum.de, s.hirche@ieee.org)

This research has been supported by CoTeSys project \#301. in the presence of non-target object [6] [7]. For example, in the experiment by Chapman and Goodale [7], the non-target object was placed at both sides of the target object in different distances. They found that the hand's reaching trajectories has a significant systematic deviation which tended away from the nearer non-target object. This result shows that the reaching trajectories can be adjusted separately for different positions of a non-target object. A possible reason comes from Sabes and Jordan's experiment [6] where the direction of the non-target object in the workspace was varied. Participants were instructed to move their finger from the start point to the target point around the non-target object. This experiment reported that there is a minimum distance between the body and the non-target object [6]. The deviation of the hand's trajectories created a larger distance to the non-target object.

In the present work we will focus on the analysis of the wrist's movement and aim to find a movement strategy for collision avoidance in the presence of a non-target object in the workspace. Another goal of this paper is to model the grasping posture of the human in the presence of an obstacle. In the robotic field, obstacle avoidance is one of the key aspects in motion planning for robotic manipulation. The model of human's grasping posture could give us a better hint to understand how humans use their redundant degrees of freedom for collision avoidance. Gerben and J. Randall [8] found that human can predict which target object would be grasped by observing the height of movement trajectories in the reach component. Therefore, we assumed the model of the grasping posture can also be used by the robot to predict human's motion and integrate the prediction into its planning for a human-robot interaction related scenario.

The paper is organized as follows. The experimental setup and results are described in Section II. The discussion of the experimental results is presented in Section III. Finally the modeling of the human's grasping posture is discussed in Section IV.

\section{EXPERIMENT}

\section{A. Experimental design}

We designed an experiment to investigate the role of the wrist movement in collision avoidance with non-target objects. We assumed that the wrist movement could be altered for different positions of a non-target object in order to 
avoid the potential collision between the body and the non-target object. Since the reach and grasp components have significant kinematics changes in the presence of the non-target object, the wrist movements connect the reach and grasp components and should also be altered in the presence of non-target objects.

\section{B. Method}

Participants: Twelve volunteers participated in the experiment: 5 male and 7 female, aged between 23 and 27 years, mean age $=24.92, \mathrm{SEM}=0.38$; mean height of shoulder (from sit position to shoulder) $=62.29 \mathrm{~cm}, \mathrm{SEM}=$ 1.52; mean length of upper arm (from shoulder to elbow) $=$ $32.83, \mathrm{SEM}=0.61$; mean length of underarm (from elbow to wrist) $=25.54, \mathrm{SEM}=0.49$; the mean length of hand (from the tip of the middle finger to the wrist) $=18.61, \mathrm{SEM}=0.27$. All participants were right-handed by self-report and were paid 8 Euro per hour for participation. All had normal or corrected-to-normal visual acuity and were naive with respect to the purpose of the study.

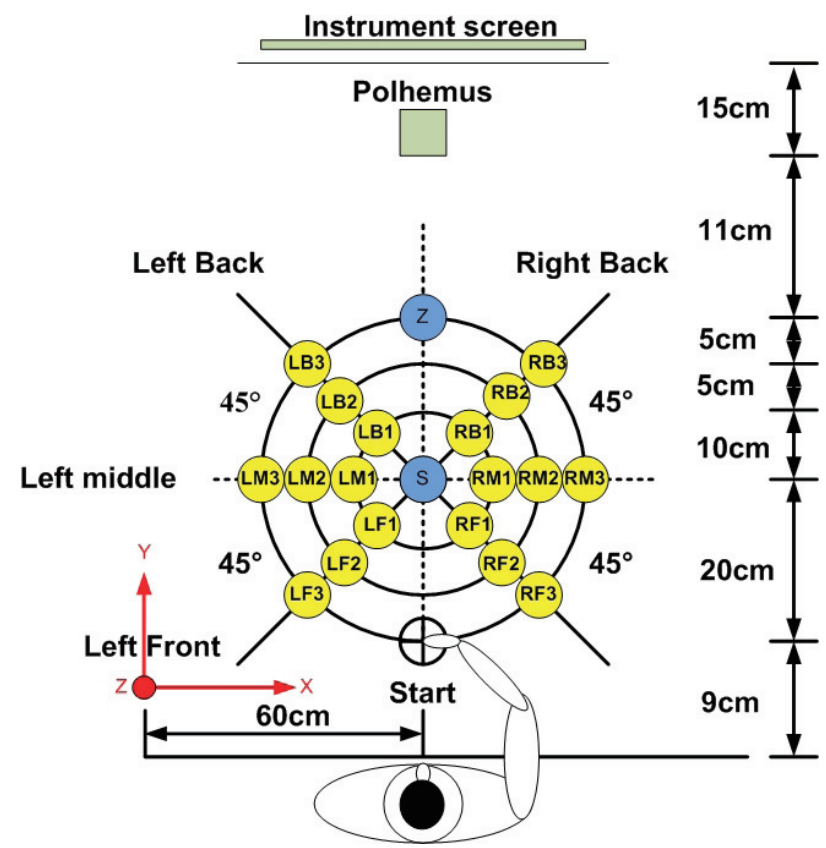

Fig.1. Schematic experimental setup showing the potential object locations on the table. The target object was placed at location ' $\mathrm{S}$ '. The non-target object could be placed at one of the yellow locations. An instruction screen was placed in front of the participant. The coordination system was defined with reference to the front-left angle of the table, the $\mathrm{x}$-axis to right, the $\mathrm{y}$-axis towards depth and the $\mathrm{z}$-axis towards height.

Apparatus: Two standard drinking glasses of the same color and size were used (top glass diameter: $7.3 \mathrm{~cm}$; diameter of bottom base: $4.5 \mathrm{~cm}$, height: $12 \mathrm{~cm}$ ). One glass was used as the target object and the other one as the non-target object. Participants sat in front of a wooden table (width: $120 \mathrm{~cm}$, depth: $75 \mathrm{~cm}$, height $73 \mathrm{~cm}$ ). Eight sensors were used to measure the respective finger-, hand- and arm movements: five sensors were attached to the finger nails of the participant's right hand, the sixth sensor was mounted at the wrist, the seventh sensor at the elbow and the eighth sensor at the shoulder. Sensor data were collected by a Polhemus Liberty magnetic motion tracker with six degrees of freedom (X, Y and $\mathrm{Z}$ coordinates, azimuth, elevation and roll and sampling rate is $240 \mathrm{~Hz}$ ). Data acquisition, experimental control and data pre-processing were done with MATLAB 2006b, statistical analyses were performed with SPSS 17.

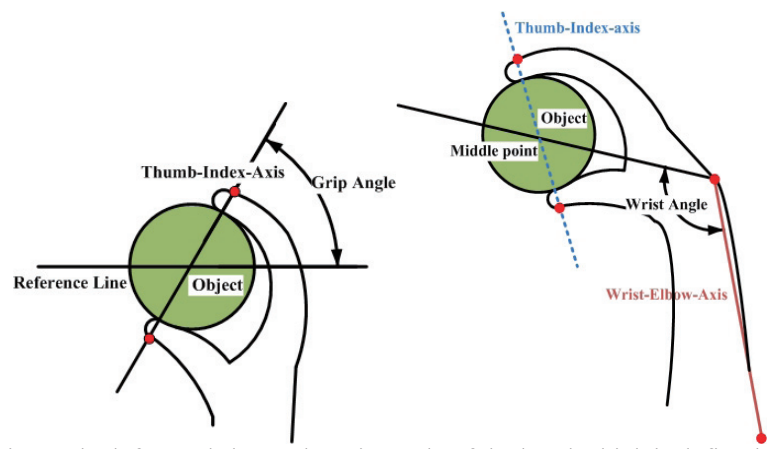

Fig.2. The left panel shows the grip angle of the hand which is defined as the angle between the line that connects the thumb and the index finger and the horizontal reference line. This angle describes the horizontal direction of the hand in the grasping manipulation. The right panel shows the wrist angle which is defined as the angle between the line that combines wrist and elbow ("Wrist-Elbow-Axis", red) and the line that connects the wrist with the centre of the target object. The wrist angle describes the relation between the arm and the hand. The red points mark the positions of sensors.

Procedure: Participants had to perform a pick-and-place task in two experimental blocks with different non-target object position conditions. The possible non-target object positions were defined in different horizontal and vertical distances to the position of the target object. Participants performed ten trials in each experimental condition. There was one non-target object (a glass) which could be placed at six locations expanding in various directions concentrically from the target pick position and three eccentricities $(10 \mathrm{~cm}$, $15 \mathrm{~cm}$ or $20 \mathrm{~cm}$ ) from the pick position ' $\mathrm{S}$ '. A trial started with the presentation of an acoustical go signal. Participants were instructed to pick the target object from the pick position ' $S$ ' and to transport it to the goal position ' $\mathrm{Z}$ ' at a comfortable speed. A trial ended when the hand of the participant returned to the start position. After each trial, the experimenter put the target object back to the pick position ' $S$ ' and placed the non-target object at a new position. All experimental conditions were presented in a randomized order to avoid that participants could predict the upcoming experimental condition.

Statistical analysis and data processing: All sensor data were filtered off-line by using a fourth-order Butterworth filter that employed a low-pass cut-off frequency of $24 \mathrm{~Hz}$. Movement trajectories were normalized by using spline interpolation. $2.55 \%$ of all trials were excluded due to errors in the experimental procedure. In addition, trials which did not satisfy the following definitions were also discarded from data analysis: (1) the grasping movement had to be started 
one second after the presentation of the beep signal; (2) the overall grasping had to be completed six seconds after the movement start; (3) each segment of the movement had to be accomplished three seconds after the last segment had finished.

Data analysis concentrated on the kinematic parameters of the wrist joint in the first movement segment. The time point when the hand left the start position and the velocity exceeded $10 \mathrm{~cm} / \mathrm{s}$ was taken as movement onset. The movement from the movement onset to the time point when the hand touched the target object with the minimal movement velocity was defined as the first movement segment. In addition, we defined two angles and a maximal deviation to describe the movement of the wrist. The grip angle (GA) was defined as the angle between the thumb-index-axis and the horizontal reference line (cf. Fig. 2, left). This angle shows the relation between the target object and the hand. The wrist angle (WA) was defined as the angle between the wrist-elbow-axis and the line which combined the wrist sensor and the middle point of the thumb-index-axis (cf. Fig. 2, right). This angle can demonstrate the relation between hand and underarm. Movement path length was defined as the 3D distance sum of all measure points. The maximal deviation was defined as the maximal 3D distance between the actual movement trajectories and the ideal movement trajectories. The ideal movement trajectory is the straight line which combines the start- and end point of every movement trajectory. Because there were diverse movements (place movement through the non-target object from the left or right side) or grasp posture (e.g. grasping with overhand or underhand), the experimental conditions in which the non-target object was placed at the point RF1-3 and RM1-3 were not considered in this analysis. The factor obstacle location therefore contained the following four levels (cf. Fig.1): right back ( $45^{\circ}$ to horizontal line) vs. left back $\left(135^{\circ}\right)$ vs. left middle $\left(180^{\circ}\right)$ vs. left front $\left(225^{\circ}\right)$. An ANOVA for the analysis of the kinematic parameters in the first movement segment was run with the within-subject factors obstacle location (as specified above) and obstacle eccentricity (near: $10 \mathrm{~cm}$ vs. middle: $15 \mathrm{~cm}$ vs. far: $20 \mathrm{~cm}$ ). The significance level was set to $\alpha=0.05$.

\section{Results}

--Grip angle and wrist angle at the pick position

The grip angle at the pick position was significantly different for the various obstacle locations (main effect 'Location', $\mathrm{F}(3,33)=44.530, \mathrm{p}<0.001)$, but not for various obstacle eccentricities (main effect 'Eccentricity', $\mathrm{p}>0.05$ ). Simultaneously there was a remarkable interaction of both factors $(F(6,66)=33.779, p<0.001)$. The grip angle was significantly larger when an obstacle was placed at the left front $\left(65.967^{\circ}, \mathrm{SEM}=4.175\right)$ than when an obstacle was placed at the left middle $\left(59.821^{\circ}, \mathrm{SEM}=3.729\right)$, the left back $\left(56.000^{\circ}, \mathrm{SEM}=3.056\right)$ or the right back $\left(37.550^{\circ}, \mathrm{SEM}=\right.$ $3.497)$. The change of the grip angle in the pick position with respect to different obstacle locations and eccentricities is shown in Fig.3a.

The wrist angle in the pick position was also significantly different only for the factor obstacle location $(\mathrm{F}(3,33)=$ $53.233, \mathrm{p}<0.001)$, but not for the factor obstacle eccentricity $(p>0.05)$. Again, there was a significant interaction of both factors $(F(6,66)=75.191, p<0.001)$. The wrist angle was significantly larger with an obstacle at the right back $\left(185.181^{\circ}, \mathrm{SEM}=2.616\right)$ than with an obstacles at the left back $\left(170.282^{\circ}, \mathrm{SEM}=2.574\right)$, the left middle $\left(166.934^{\circ}\right.$, $\mathrm{SEM}=2.876)$ and the left front $\left(162.010^{\circ}, \mathrm{SEM}=3.346\right)$. Fig. $3 \mathrm{~b}$ shows the change of the wrist angle in the pick position for different obstacle positions and eccentricities.

\section{$3 \mathrm{a}$}

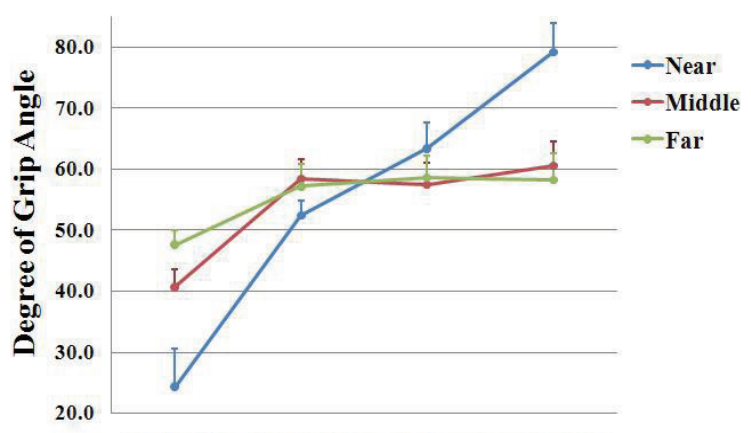

Right Back Left Back Left Middle Left Front

$3 b$

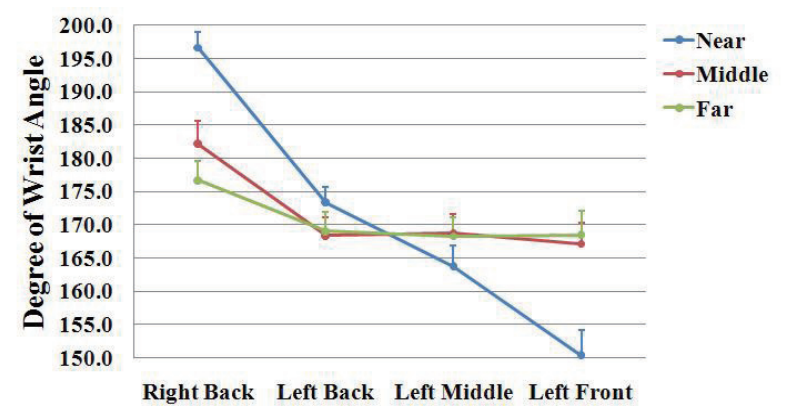

Fig.3. Change of grip angle (upper panel) and wrist angle (lower panel) for different obstacle positions. Locations are marked at the horizontal axis; eccentricities are visualized as various line colours.

\section{--Kinematics parameters}

The mean wrist path length in the first movement segment differed significantly for obstacles at various locations $(\mathrm{F}(3,33)=29.731, \mathrm{p}<0.001)$ and eccentricities $(\mathrm{F}(2,22)=$ $5.893, \mathrm{p}<0.009)$; also the interaction of both factors reached significance $(\mathrm{F}(6,66)=13.033, \mathrm{p}<0.001)$. Wrist movements were longer when an obstacle was placed at the left front $(27.550 \mathrm{~cm}, \mathrm{SEM}=0.918)$ than for an obstacle at the left middle $(26.110 \mathrm{~cm}, \mathrm{SEM}=0.793)$, the left back $(25.441 \mathrm{~cm}$, $\mathrm{SEM}=0.614)$ and the right back $(23.300 \mathrm{~cm}, \mathrm{SEM}=0.424)$. Additionally, the movement trajectories of the wrist were longer for obstacles at near eccentricities $(25.983 \mathrm{~cm}, \mathrm{SEM}=$ 
0.748) compared to obstacles at middle eccentricities $(25.427 \mathrm{~cm}, \mathrm{SEM}=0.659)$ and far eccentricities $(25.391 \mathrm{~cm}$, $\mathrm{SEM}=0.572)$.

The mean maximal deviation of the wrist in the first segment was also significantly different for obstacles at various locations $(\mathrm{F}(3,33)=15.854, \mathrm{p}<0.001)$, but not for various eccentricities $(\mathrm{p}>0.05)$. Simultaneously there was a significant interaction of both factors $(\mathrm{F}(6,66)=6.804, \mathrm{p}<$ $0.001)$. Wrist movements had a larger maximum deviation
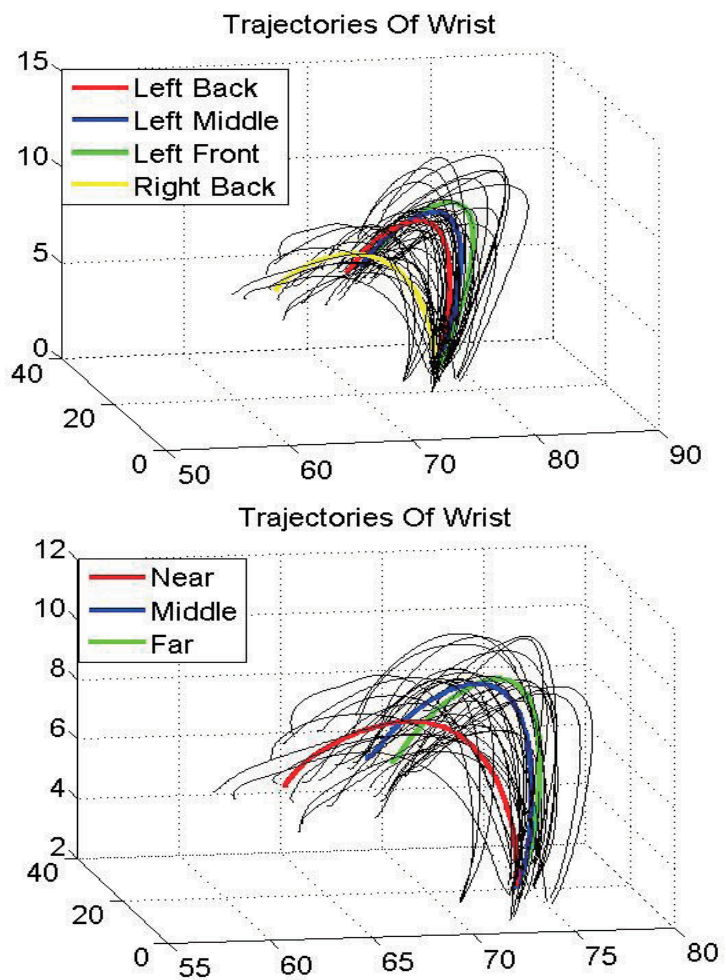

Fig.4. Movement trajectories of the wrist. Upper panel: Movement trajectories of the wrist for obstacles at different locations but fixed eccentricity $(10 \mathrm{~cm})$. Lower panel: Movement trajectories of the wrist for obstacles at different eccentricities but fixed location (right back). The black lines show the movement trajectories of trials averaged across all participants; the coloured lines show the grand average of the respective factors.

with an obstacle at the left front $(4.363 \mathrm{~cm}, \mathrm{SEM}=0.207)$ compared to the left middle $(4.272 \mathrm{~cm}, \mathrm{SEM}=0.206)$, the left back $(4.229 \mathrm{~cm}, \mathrm{SEM}=0.218)$ and the right back $(3.497 \mathrm{~cm}$, $\mathrm{SEM}=0.148$ ). Movement trajectories of the wrist are shown in Figure 4. Means of all parameters observed in the first movement segment are given in Table.1.

\section{DISCUSSION}

Results showed that when humans are grasping an object, both grip angle and wrist angle at the pick position can be modified with respect to different obstacle positions. These results show that in the movement plan "collision avoidance" humans also take the grasping direction and the position of potential obstacles into account before they adjust their wrist joint. Humans grasp the farther sides of the target object in relation to the non-target object. These Results strengthen Willemijn and Jeroen's experimental discovery [9]. In their experiment, it is found that human adjusted the grasping direction with respect to the object's position. Our results proposed further that the grasping direction depends not only on the location of the target object, but also on the location of the non-target object. The specification of the wrist movement and the rotation of the wrist joint might be one of the human strategies to avoid the potential collision between the arm and a possible non-target object.

Results showed that the mean path length of the wrist movement was significantly affected by the location of the non-target, distracting object. Both the horizontal and the vertical distance from the target object were taken into account. However, the maximum deviation of the wrist movement differed only for the vertical obstacle position and not for the (horizontal) obstacle eccentricity. The analysis showed that the increase of the mean movement path was due to the rotation of the wrist joint. Moreover, the analysis of the maximum deviation of the movement trajectories showed that the increase of path length was accompanied by a greater movement deviation of the wrist joint during the prehensile movement. Concerning the vertical distance from the target object, the mean path lengths numerically differ, but there was no significant, that is, systematic deviation. This may be due to the fact that the rotation of the wrist joint depended on the obstacle's relative position and was flexibly chosen for various conditions. For example, with an obstacle at the right back, the rotation of the wrist was counterclockwise with increasing eccentricity, while for an obstacle at the left front the rotation of the wrist was clockwise. These results show that the flexibility of the wrist movement provides more possibilities to avoid possible collisions.

\section{HUMAN'S WRIST MOVEMENT MODEL}

In this section, the model of the human's wrist movement is proposed which could help us to understand better the collision avoidance mechanism in the human. Many strategies have been proposed for the collision avoidance in robotics, for example the potential function based approach [10]. Thus, one interesting question is how humans use their redundant degrees of freedom for collision avoidance. Furthermore, the model of human's wrist movement can also be used to predict human's motion. The prediction can then be used in the robot's planning for a human-robot interaction scenario. In this paper, as a first step towards the modelling, the influence of the obstacle position in relation to the target object has been analyzed and a preliminary model is proposed. 
TABLE I

The Mean value of kinematics parameters

\begin{tabular}{cccccc}
\hline \hline $\begin{array}{c}\text { Non-Target (Obstacle) Factors } \\
\text { Obstacle Location } \\
\text { (4 Levels) }\end{array}$ & $\begin{array}{c}\text { Eccentricity } \\
\text { (3 Levels) }\end{array}$ & Path(cm) & Deviation(cm) & GA(degree) & WA(degree) \\
\hline \multirow{2}{*}{ Right Back } & $10 \mathrm{~cm}$ & $22.539(0.408)$ & $2.909(0.240)$ & $24.362(6.243)$ & $196.64(2.386)$ \\
& $15 \mathrm{~cm}$ & $23.414(0.535)$ & $3.573(0.171)$ & $40.69(2.986)$ & $182.219(3.364)$ \\
& $20 \mathrm{~cm}$ & $23.946(0.436)$ & $4.01(0.236)$ & $47.597(2.315)$ & $176.685(2.934)$ \\
& $10 \mathrm{~cm}$ & $24.715(0.537)$ & $4.071(0.214)$ & $52.427(2.480)$ & $173.381(2.307)$ \\
Left Back & $15 \mathrm{~cm}$ & $25.787(0.664)$ & $4.301(0.244)$ & $58.395(3.248)$ & $168.403(2.707)$ \\
& $20 \mathrm{~cm}$ & $25.82(0.693)$ & $4.316(0.226)$ & $57.176(3.667)$ & $169.061(2.933)$ \\
& $10 \mathrm{~cm}$ & $27.056(1.15)$ & $4.437(0.259)$ & $63.422(4.133)$ & $163.771(3.047)$ \\
Left Middle & $15 \mathrm{~cm}$ & $25.768(0.656)$ & $4.198(0.203)$ & $57.443(3.64)$ & $168.77(2.884)$ \\
& $20 \mathrm{~cm}$ & $25.507(0.652)$ & $4.181(0.22)$ & $58.598(3.595)$ & $168.26(2.833)$ \\
& $10 \mathrm{~cm}$ & $29.622(1.151)$ & $4.468(0.217)$ & $79.144(4.752)$ & $150.397(3.725)$ \\
Left Front & $15 \mathrm{~cm}$ & $26.739(0.995)$ & $4.369(0.266)$ & $60.54(4.011)$ & $167.127(3.157)$ \\
& $20 \mathrm{~cm}$ & $26.289(0.751)$ & $4.253(0.182)$ & $58.217(4.294)$ & $168.505(3.571)$ \\
\hline \hline
\end{tabular}

Means observed in the first movement segment, standard error of means are given in brackets. Movement path and maximal deviation of the movement are given in $\mathbf{c m}$. Grip angle (GA) and wrist angle (WA) are given in degree $\left({ }^{\circ}\right)$. The first two letters indicate the obstacle location $(\boldsymbol{R B}$ : right back; $\boldsymbol{L B}$ : left back; $\boldsymbol{L M}$ : left middle; $\boldsymbol{L F}$ : left front). The last number indicates the obstacle eccentricity $(\boldsymbol{I}:$ near $(10 \mathrm{~cm})$; 2: middle (15cm); 3: far (20)).

It is assumed that the coupling between the wrist angle and the grip angle is sufficiently small and can therefore be neglected. In order to model the grasping posture, we reconsider the changes of the posture as the eccentricity is increased while the obstacle location is fixed. Moreover, since the experiment is conducted for a limited number of obstacle eccentricities and locations, first the results are

$5 \mathrm{a}$

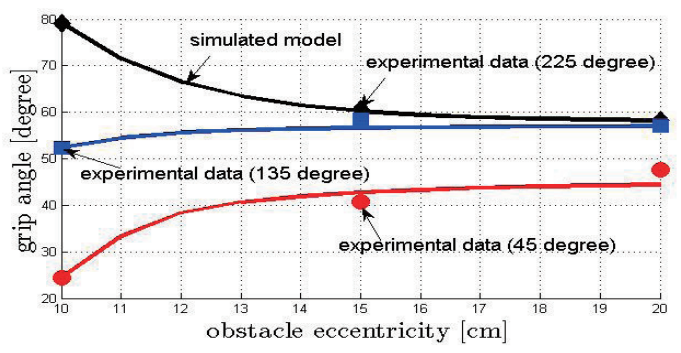

$5 b$

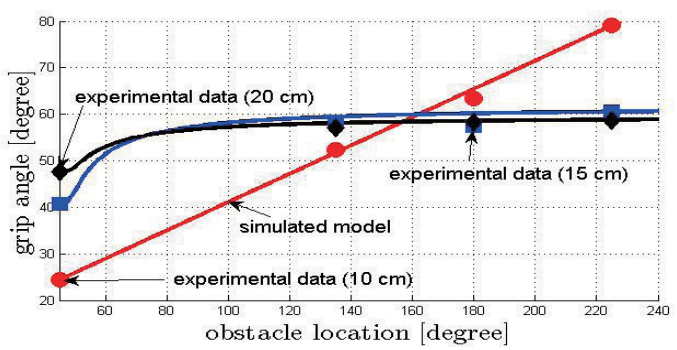

Fig.5. 5a: The change of the grip angle with respect to the eccentricity for a fixed location (diamond: 225 degree, rectangle: 135 degree, circle: 45 degree); 5 b: the change of the grip angle with respect to the location for a fixed eccentricity (diamond: $20 \mathrm{~cm}$, rectangle: $15 \mathrm{~cm}$, circle: $10 \mathrm{~cm}$ ). The solid line shows the results simulated from the model. interpolated in order to have a smooth curve. The results are shown in Fig. 5a and Fig. 6a for the grip and wrist angle respectively. As can be observed from the figures, there is a limit for the obstacle eccentricity $\left(d_{c}=15 \mathrm{~cm}\right)$ beyond which the wrist and grip angle did not change i.e. the non-target object did not affect the posture. Moreover, the changes of the grasping posture are reconsidered in a case in which the obstacle location was varied between $45^{\circ}$ and $225^{\circ}$ while the eccentricity was kept constant. The results are shown in Fig. $5 \mathrm{~b}$ and in Fig. $6 \mathrm{~b}$ for the grip and wrist angle respectively. As can be observed from Fig. 5b and Fig. 6b, if the obstacle eccentricity is larger than the limit i.e. $d>d_{c}$, then there exists a limit for the obstacle location $\left(\theta_{c}=120^{\circ}\right)$ in which the grasping posture does not change. Furthermore, as shown in Fig. 5a and Fig. 6a, depending on the obstacle location, i.e., on the left or on the right of the object, the trajectories will converge to different values even though the obstacle is far from the object, i.e., $d>d_{c}$. This is possibly due to the flexibility of the grasping posture needed for the planning of the reaching movement by the human.

Based on these facts, the following model for the grasping posture and the wrist angle are proposed. As can be observed from Fig. 5a and Fig. 6a, for both cases, i.e. when the obstacle is on the right side $\left(45^{\circ}<\theta<90^{\circ}\right)$ and left side $\left(90^{\circ}<\theta<225^{\circ}\right)$, the wrist and grip angle can be approximated by an exponential function given by

$$
\begin{aligned}
& \theta_{w}=A_{w} e^{x}+\theta_{w_{o}} \\
& \theta_{g}=A_{g} e^{y}+\theta_{g_{o}}
\end{aligned}
$$


TABLE II

Parameters for simulated model

\begin{tabular}{cccccccc}
\hline \hline Grip angle & $A_{g}$ & $a_{g}$ & $\theta_{g a}$ & Grip angle & $A_{g}$ & $a_{g}$ & $\theta_{g a}$ \\
$225^{\circ}$ & 21.14 & -0.45 & 58 & $10 \mathrm{~cm}$ & - & - & - \\
$135^{\circ}$ & 5 & -0.9 & 52 & $15 \mathrm{~cm}$ & 21 & -10.1 & 40.69 \\
$45^{\circ}$ & 22 & -0.9 & 24.36 & $20 \mathrm{~cm}$ & 12 & -12 & 47.6 \\
\hline Wrist angle & $A_{w}$ & $a_{w}$ & $\theta_{w a}$ & Wrist angle & $A_{w}$ & $a_{w}$ & $\theta_{w a}$ \\
$225^{\circ}$ & 20 & -0.9 & 150.39 & $10 \mathrm{~cm}$ & - & - & - \\
$135^{\circ}$ & 4.38 & -0.6 & 169 & $15 \mathrm{~cm}$ & 14.22 & -0.2 & 168 \\
$45^{\circ}$ & 18.63 & -0.3 & 178 & $20 \mathrm{~cm}$ & 7.68 & -0.3 & 169 \\
\hline \hline
\end{tabular}

where $\theta_{w}, \theta_{g}$ are the wrist angle and grip angle respectively. The parameter $x$ (resp. $y$ ) is defined as $x=a_{w} d$ or $x=a_{w} / d$ (resp. $y=a_{g} d$ or $y=a_{g} / d$ ) depending on the object location. $d$ is the object eccentricity, $a_{w}$ and $a_{g}$ are the decay rates, and $\theta_{w a}$, $\theta_{g a}$ are the wrist and grip angle when there are no obstacles in the environment i.e. when $d>d_{c}$ for $x=a_{w} d$ or $y=a_{g} d$ and $A w$, $\mathrm{Ag}$ are constant. A similar model to (1), (2) can also be used to model the grip and wrist angle w.r.t. the object location. Moreover, as can be seen from Fig. 5b and Fig. 6b when the eccentricity $d<d_{c}, \boldsymbol{A}_{w}$ (resp. $\boldsymbol{A}_{g}$ ) is inversely proportional (resp. directly proportional) to the increase of location. Thus, from this relationship, the $A_{w}$ and $A g$ for every location can be computed. As can be observed from Fig. 5a and Fig. 6a, the decay rates of $a_{w}$ and $a_{g}$ are depending on the obstacle location. Using the model in (1) and (2), the grasping posture for a given obstacle position can be predicted. Next, we simulate the wrist movement based on the proposed model with parameters given in Table II. It can be observed from Fig.5 and Fig. 6 that the proposed model could approximate the experimental wrist movement. Moreover, as can be observed from the model and the experimental data, humans use more of their wrist when there is an obstacle nearby than

$6 a$

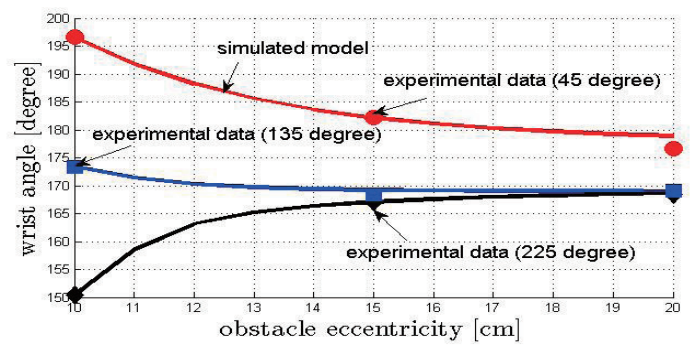

$6 b$

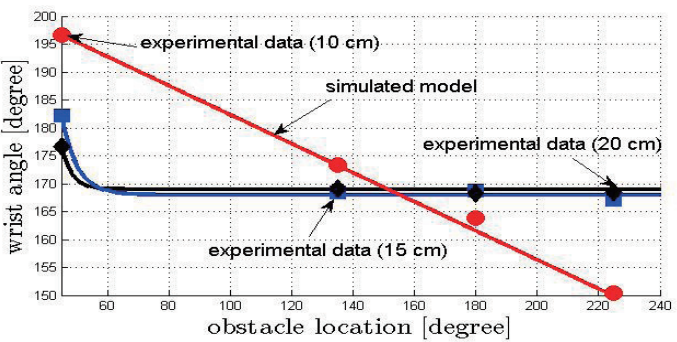

Fig.6. 6a: The change of the wrist angle with respect to the eccentricity for a fixed location (diamond: 225 degree, rectangle: 135 degree, circle line: 45 degree); $6 \mathrm{~b}$ : the change of the wrist angle with respect to the location for a fixed eccentricity (diamond: $20 \mathrm{~cm}$, rectangle: $15 \mathrm{~cm}$, circle: $10 \mathrm{~cm}$ ). The solid line shows the results simulated from the model when it is far from their hand. This gives a preliminary result to understand how humans use their degrees of freedom for collision avoidance. In the future, the dynamics of the decay rate in (1) and (2) will be derived.

\section{CONCLUSION}

In this paper, the important role of wrist movements for avoidance of a non-target object in a pick-and-place experiment is investigated. It is shown that humans adjust the position of their wrist and the angle between their underarm and hand relative to the position of a non-target object. Moreover, humans tend to grasp the target object from the side which is further from the non-target object. In addition, a preliminary model of the wrist movement is proposed which can be used to predict the human motion for a robotic control. It can also be observed from the model that humans use more of their wrist when there is an obstacle nearby. In the future, the coupling between the wrist and grip angle will be investigated in order to have a better model of the wrist movement.

\section{REFERENCES}

[1] M. Jeannerod, "Intersegmental coordination during reaching at natural visual objects," Attention and Performance IX, 1981, pp. 153-169.

[2] J.R. Tresilian, "Attention in action or obstruction of movement? A kinematic analysis of avoidance behavior in prehension.," Experimental brain research., vol. 120, 1998, pp. 352-68.

[3] M. Mon-Williams, J.R. Tresilian, V.L. Coppard, and R.G. Carson, "The effect of obstacle position on reach-to-grasp movements," Experimental Brain Research, vol. 137, 2001, pp. 497-501.

[4] M. Biegstraaten, J.B. Smeets, and E. Brenner, "The influence of obstacles on the speed of grasping.," Experimental brain research Experimentelle Hirnforschung. Expérimentation cérébrale, vol. 149, 2003, pp. 530-4.

[5] M. Saling, J. Alberts, G.E. Stelmach, and J.R. Bloedel, "Reach-to-grasp movements during obstacle avoidance," Experimental Brain Research, vol. 118, 1998, pp. 251-258.

[6] P.N. Sabes and M.I. Jordan, "Obstacle Avoidance and a Perturbation Sensitivity Model for Motor Planning," Journal of Neuroscience, vol. 17, 1997, pp. 7119-7128.

C.S. Chapman and M.a. Goodale, "Missing in action: the effect of obstacle position and size on avoidance while reaching.," Experimental brain research. Experimentelle Hirnforschung. Expérimentation cérébrale, vol. 191, 2008, pp. 83-97.

[8] G. Rotman, N.F. Troje, R.S. Johansson, and J.R. Flanagan, "Eye movements when observing predictable and unpredictable actions.," Journal of neurophysiology, vol. 96, 2006, pp. 1358-69. W.D. Schot, E. Brenner, and J.B. Smeets, "Posture of the arm when grasping spheres to place them elsewhere.," Experimental brain research. Experimentelle Hirnforschung. Expérimentation cérébrale, vol. 204, 2010, pp. 163-71.

[10] M. Spong, S. Hutchinson and M. Vidyasagar, Robot Modeling and Control, John Wiley \& Sons, Inc, 2006 